\title{
Quantitative clonal barcode analysis for the identification of key drivers of disease progression and drug resistance Cellecta (Mountain View, CA, USA)
}

The ability to label and trace individual cells is a powerful experimental approach in many research areas, including stem cell biology, evolution and cancer biology. Labeling of individual cancer cells with different engineered or natural driver lesions and modeling of in vivo or in vitro clonal expansion can facilitate discovery of genetic determinants of increased pathogenicity, cancer progression, or drug resistance.

CloneTracker XPTM Barcode Libraries are the newest genetic tool for high-resolution viral genetic barcoding. When combined with nextgeneration sequencing (NGS) analysis, they enable tracking and quantitation of the proliferation of single cells in a heterogeneous cell population. The lentiviral CloneTracker XP libraries have two key functional elements: reporter and drug resistance markers expressed from a constitutive EFS promoter, and a barcoded cassette (Figure 1).

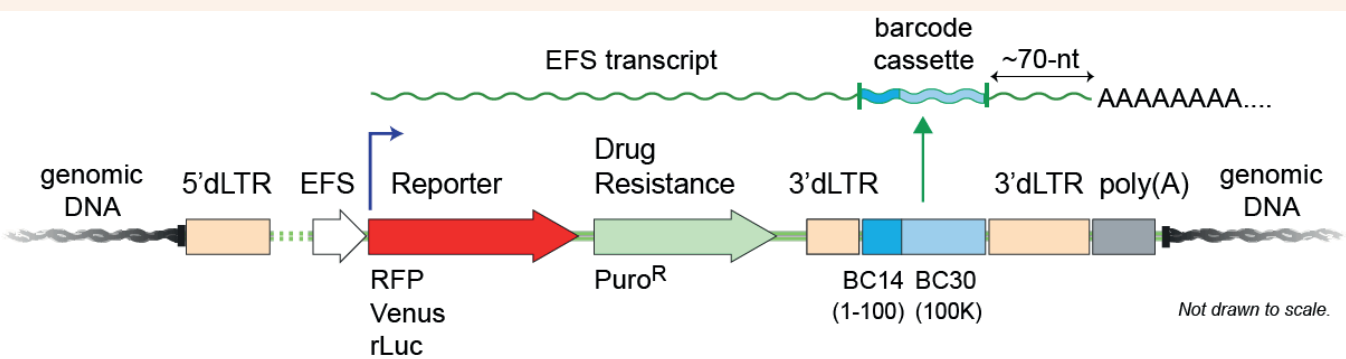

Figure 1. Design of CloneTracker XP lentiviral libraries for genetic barcoding and clonal analysis at the single-cell level.

FACS analysis (e.g., for HCS differentiation models). Red-emitting luciferase (rLuc) is the most sensitive bioluminescence reporter currently available for in vivo imaging applications and even detects metastatic lesions in mouse cancer models (Branchini BR et al. Red-emitting luciferases for bioluminescence reporter and imaging applications. Anal. Biochem. [2010] 396, 290-297).

The barcoded cassette located in the 3'-dLTR region has a composite structure built from 14-n barcodes (from 1 to 100 different sequences) and 30-n barcodes (100,000 different sequences). Each composite (14n-30n) barcode has a unique sequence that tolerates up to five mutations and could be easily distinguished even in the most challenging applications (e.g., in a mouse metastatic model) from the mutational background introduced by extended cell growth or NGS analysis. 14-n barcodes allow labeling of up to 100 - in the current CloneTracker XP version up to 10 - different cell populations with unique barcodes (e.g., with different genetic backgrounds or engineered cells using a CRISPR system). It also allows use of mixes of these cells in the tumor growth assay. Furthermore, the 30-n barcodes allow labeling of individual cells with unique barcodes in each defined cell pool and quantitative monitoring of the proliferation of each clone.

Importantly, the composite barcode cassette is transcribed from the EFS promoter and located in the 3'-UTR region of the reporter transcript and thus can be amplified from both genomic DNA or cDNA of barcoded cells. The transcriptomes of cell clones labeled with specific barcodes could be further analyzed for gene expression phenotyping using commonly used single-cell analysis technologies (e.g., DropSeq, 10x Genomics, BD Rhapsody, Takara SMARTer ICELL8 systems). Transcribed barcodes allow the correlation of each expression profile to the specific clones derived from progenitor cells with a defined genetic background.

Quantitative clonal barcode analysis in the mixes of genetically engineered cells grown in vitro (e.g., organoids) or in in vivo mouse models offers the unique research strategy of uncovering key drivers for tumor initiation, progression and resistance to therapeutic drugs.

More information is available at www.cellecta.com/CloneTrackerXP

Biotechniques 65: 169 (September 2018) doi: 10.2144/btn-2018-2002

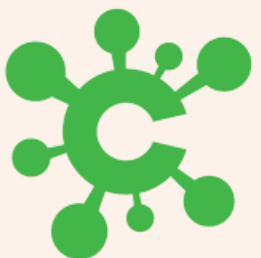

CELLECTA 